\title{
Tuning the Lateral Leakage Loss of TM-like modes in Shallow-etched Waveguides using Liquid Crystals
}

\author{
Thomas Ako, ${ }^{1,2, *}$ Jeroen Beeckman, ${ }^{1,2}$ Wim Bogaerts, ${ }^{2,3}$ and Kristiaan Neyts ${ }^{1,2}$ \\ ${ }^{1}$ Liquid Crystals and Photonics Group, Department of Electronics and Information Systems, \\ Ghent University, St. Pietersnieuwstraat 41, 9000 Ghent, Belgium \\ ${ }^{2}$ Center for Nano- and Bio-Photonics, Ghent University, \\ St. Pietersnieuwstraat 41, 9000 Ghent, Belgium \\ ${ }^{3}$ Photonics Research Group, Department of Information Technology, \\ Ghent University, St. Pietersnieuwstraat 41, 9000 Ghent, Belgium
}

compiled: November 8, 2013

\begin{abstract}
We examine the tuning effect a liquid crystal cladding has on the lateral leakage loss of TM-like modes in shallow-etched waveguides. For such waveguides with an air cladding, the guided TM-like mode and the unguided cladding TE-like mode can only be phase matched at precisely one angle. We find that for an anisotropic cladding such as a liquid crystal, this phase matching is now possible for a range of angles. Each of these angles corresponds to a given orientation of the molecules in the liquid crystal cladding. We show that the waveguide width at which the minimum in leakage loss occurs can be changed by varying the orientation of the liquid crystal cladding. We find different tuning regimes, identify a suitable tuning range and discuss the feasibility of tunable leakage loss experiments.
\end{abstract}

OCIS codes: $\quad 230.3120,230.2090,230.7390,130.4815$.

http://dx.doi.org/10.1364/XX.99.099999

\section{Introduction}

Silicon-on-insulator (SOI) waveguides possess many desirable properties which make them suitable for highdensity integration on a single chip. SOI technology has been very successful because it utilises a high index contrast material system [1] and is compatible with complementary metal-oxide-semiconductor (CMOS) fabrication technology (i.e. it is cheap). An interesting class of SOI waveguide is the quasi planar shallow-etched waveguide [2-4] which is suitable for active SOI devices like modulators and/or light-emitting sources. Unfortunately, for certain practical geometries, the TM-like mode of these waveguides exhibits lateral leakage loss. The effective index of the guided TM-like mode of the waveguide is less than that of the radiating (cladding) slab TE-like mode. This slab TE-like mode can propagate in any direction since it is unguided and can be phase matched to the guided TM-like mode for a particular angle. Consequently, the guided TM mode suffers from lateral leakage loss since it can be phase matched to a radiating mode.

Recently in the literature, the existence of this leakage loss and its dramatic dependence on the width of the waveguide has been observed experimentally [5]

\footnotetext{
* Corresponding author: thomas.ako@elis.ugent.be
}

for straight waveguides. In [6], lateral leakage loss in shallow-etched bent waveguides is analyzed. Here the lateral leakage is shown to depend on both the bend radius and the waveguide width. In other studies $[7,8]$ a waveguide structure with a dimple at its center is proposed. It is then shown that such a structure is both low loss and fabrication tolerant. [9] proposes a structure wherein two waveguides are parallel to each other. Depending on the gap between the two waveguides, the leakage loss can be cancelled when both waveguides are excited with quadrature phase.

So far, previous studies have been limited in terms of controlling the lateral leakage loss in shallow-etched waveguides. For a waveguide at a constant temperature, with no applied voltage and no stress; the effective indices of each of the modes in the waveguide are fixed. The leakage properties of the waveguide are then also fixed since they arise from the interplay between the effective indices. If we can modulate the effective indices of the waveguide modes, then we would also modulate the leakage loss of the waveguide. In practice this can be done by thermo-optics or electro-optics. In silicon, the thermo-optic effect is large enough for practical applications compared to the the electro-optic effect [10]. Consequently, temperature tuning is often considered for tuning SOI waveguides [11], but this requires constant power supply and the tuning range is limited. A material 
with a high electro-optic effect can be integrated with silicon in order to tune it electro-optically. An example of such a material is a liquid crystal (LC). LCs have excellent electro-optic properties. Their large electro-optic coefficient has established them as the material of choice in display applications. However, they are also suitable for non-display applications. They have been used for different tunable photonic components like spatial light modulators, lasers, filters, switches and nonlinear components [12]. Many groups have proposed designs in which LCs are used to alter the properties of waveguides. The LC can be used as a tunable cladding material [1315] or as the guiding medium [16-18].

We propose a structure in which a nematic LC layer is used as the upper cladding of a conventional shallowetched waveguide. The orientation of the LC in the cladding can be changed by the application of a moderate voltage. This change in orientation is accompanied by a change in the cladding index [14] felt by the modes in the waveguide and results in a change in the effective index of these modes. Since the lateral leakage loss arises from the interplay of these effective indices, this causes a change in the leakage loss of the waveguide.

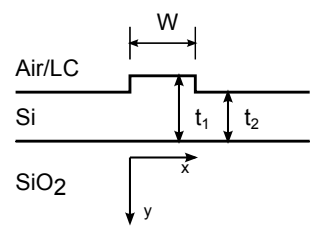

Fig. 1. Problem geometry, the positive z-direction is into the plane of the page.

\section{Lateral Leakage Loss Mechanism}

Before proceeding any further a brief review of the mechanism for lateral leakage loss is in order. The optical fields in an SOI shallow-etched waveguide are confined in the y-direction by the index mismatch between air, silicon and silica (See fig. 1). In the x-direction, confinement is provided by the step discontinuity in silicon. As it travels along the waveguide, the TE-like (resp. TMlike) mode gets additional small $H_{x}$ and $E_{z}$ (resp. $E_{x}$ and $H_{z}$ ) components at the waveguide boundaries [19]. Consequently, we have TE-like and TM-like modes in such waveguides; with TE-TM (and TM-TE) mode conversion occurring at the waveguide walls. Mode conversion can lead to lateral leakage loss if certain conditions are satisfied. In each case, we must examine the graph in fig. 2 to determine which modes are evanescent in the cladding and hence determine which mode suffers from lateral leakage loss.

For a TE-like propagating shallow-etched waveguide mode, the TE field must necessarily be laterally evanescent in the shallow-etched waveguide cladding. In general, the propagation constant of the TM-like mode in a slab waveguide with thickness $t_{2}$ is lower than that of the TE-like mode in the waveguide. Hence TM fields resulting from TE-TM mode conversion are evanescent. Consequently, the TE-like modes show no lateral leakage loss.

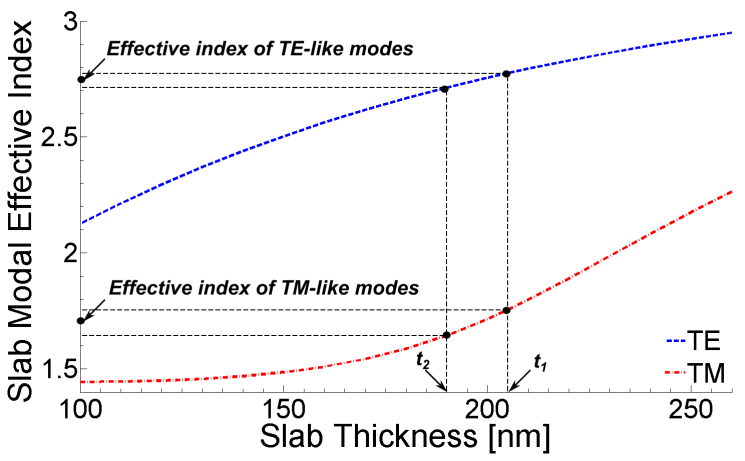

Fig. 2. Variation of effective index of the fundamental TE and TM modes with slab thickness for an SOI slab waveguide with air upper cladding. The plot is obtained using a fully vectorial finite element solver; the operating wavelength is $1550 \mathrm{~nm}$.

For a TM-like propagating shallow-etched waveguide mode, the TM field in the cladding must be evanescent as well. However, the propagation constant of the TElike mode in a slab waveguide with thickness $t_{2}$ is larger than that of the TM-like mode in the waveguide. Hence TE fields which arise due to TM-TE conversion at the ridge boundary are not evanescent. These TE fields can be phase matched to a TE slab mode propagating laterally at angle $\theta$ in the slab region [5]. Consequently, the TM-like mode in these waveguides exhibits lateral leakage loss.

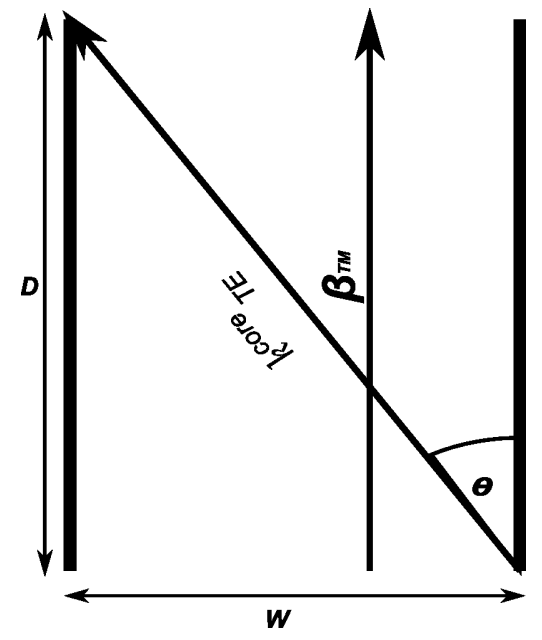

Fig. 3. Top view of the waveguide. The TM mode travels along the waveguide, we consider one of the TE fields it generates at the ridge wall.

Consider a TM-like mode propagating in a shallowetched waveguide (see Fig. 3). At the right waveguide boundary, it generates transmitted and reflected 
TE waves which have a relative phase of $\pi$. The reflected TE wave travels through the waveguide core at an angle to the $\mathrm{z}$ axis since it is not guided. When it gets to the left waveguide boundary, the guided TM-like mode again generates transmitted and reflected $\mathrm{TE}$ waves with a relative phase of $\pi$. The new transmitted TE wave combines with the previously reflected TE wave. If the phase accumulated by the previously reflected TE wave as it travels through the waveguide core is a multiple of $2 \pi$, then it will interfere destructively with the newly transmitted TE wave. We can write this condition as follows;

$$
\beta_{T M} \cdot D-k_{T E}^{(\text {core })} \cdot \sqrt{D^{2}+W^{2}}=2 m \pi .
$$

Where $\mathrm{m}$ is a positive integer. After some simple algebra, we arrive at the well known expression for the resonance condition of the leakage loss of SOI shallowetched waveguides.

$$
W=\frac{m \lambda}{\sqrt{n_{e f f, T E}^{(c o r e)}{ }^{2}-N_{e f f, T M^{2}}}}
$$

Where $\lambda$ is the wavelength of the light in the waveguide, $N_{\text {eff,TM }}$ is the effective index of the guided TMlike waveguide mode and $n_{e f f, T E}^{(\text {core }}$ is the effective index of the (unguided) TE wave which traverses the waveguide core.

\section{Tuning the Lateral Leakage Loss of the TM-like mode}

For a shallow-etched waveguide with air cladding [5-9], the TE and TM mode feel the same refractive index in the cladding. When LC is used as the upper cladding layer, the situation becomes far more interesting. The LC can be oriented such that the TE and TM modes in the waveguide feel a different refractive index in the cladding [14]. In this way it is possible to change the effective index of the TE and TM modes separately; this is equivalent to changing $n_{e f f, T E}^{(c o r e)}$ and/or $N_{e f f, T M}$ in (2) from the values they had when the waveguide had an air cladding. If this is the case, the resonance condition can be fulfilled if $W=W_{L C}$ which will in general be different from $W_{\text {air }}$.

There are three main possibilities for orienting the LC in the cladding. First, orient the $\mathrm{LC}$ along the $\mathrm{x}$-axis; the TE and TM modes feel the extraordinary $\left(n_{e}\right)$ and ordinary $\left(n_{o}\right)$ indices of the LC in the cladding respectively. Second, we could orient the LC along the y-axis; the TE and TM modes would feel an index $n_{o}$ and $n_{e}$ in the cladding respectively. Third, we could orient the LC along the z-axis. In this case, both modes only feel the ordinary index $\left(n_{o}\right)$ of the LC in the cladding. We can choose any of the three states above as the initial state of the LC and then choose one from the remaining two as the final state of the LC. We choose an LC layer as in options 1 and 2 above as the initial and final states. For this combination we would see the largest change in the effective indices and hence leakage loss of the waveguide.

It is instructive to take a look at the k-vector diagram for a waveguide with an anisotropic upper cladding. Fig. 4 shows k-vector diagrams of the fundamental $\mathrm{TE}$ and TM modes and the radiating cladding TE mode; for an air cladding and for claddings as in options 1 and 2 above. Given that the TE and TM modes feel an index $n_{o}$ or $n_{e}$ depending on the LC alignment, we can make the following observations. The propagation constant of the TM mode in (c) is greater than that in (b). On the other hand, the propagation constant of the TE mode in (b) is greater than that in (c). Finally, the unguided TE slab mode propagation constant $\left(k_{T E}^{(\text {clad })}\right)$ is not the same in every direction for a cladding made out of $\mathrm{LC}$ aligned along the $\mathrm{x}$-axis. In this case the refractive index felt by this mode in the cladding is given by;

$$
n(\theta)=\frac{1}{\sqrt{\left(\frac{\cos \theta}{n_{e}}\right)^{2}+\left(\frac{\sin \theta}{n_{o}}\right)^{2}}}
$$

In (b) the tip of $k_{T E}^{(\text {clad) }}$ traces out a quadrant of an ellipse rather than that of a circle $((\mathrm{a}),(\mathrm{c}))$. It is this anisotropy in cladding index that makes the tuning possible. In previous studies with an air cladding, $\beta_{T M}$ and $k_{T E}^{(\text {clad })}$ can only be phase matched at precisely one angle. When an LC cladding is used, they can now be phase matched for a range of angles. Each of these angles corresponds to a given orientation of the LC in the cladding. By varying the orientation of the $\mathrm{LC}$ in the cladding, it is then possible to vary the waveguide width at which the minima in leakage loss occur.

In order to confirm the qualitative arguments given above, we consider two waveguide geometries; (i) $t_{1}=$ $205 \mathrm{~nm}, t_{2}=190 \mathrm{~nm}$ and (ii) $t_{1}=220 \mathrm{~nm}, t_{2}=150 \mathrm{~nm}$. For each of these geometries, we calculate the effective index of the TM mode for waveguide widths between $500 \mathrm{~nm}$ and $2500 \mathrm{~nm}$. We also calculate the effective index the TE mode in a slab waveguide with thickness $t_{1}$. We do this for the three cases in fig. 4. The operating wavelength is $1.55 \mu \mathrm{m}$ and we only consider the fundamental waveguide modes. The refractive indices of the core and cladding of the waveguide are $n_{\text {core }}=3.475$ and $n_{\text {clad }}=1.444$ respectively. For the LC upper cladding, we use the LC E7 $\left(n_{e}=1.7, n_{o}=1.5\right.$ for a wavelength of $1.55 \mu \mathrm{m}$ ). For claddings as in (a) and (c) in fig.4, there is no need to take into account the direction of the unguided TE wave in the waveguide core. For a cladding as in (b) in fig.4, the calculation is more involved. We first compute the effective index of the TM mode for each waveguide width. Next, we obtain the effective index of the TE mode in a slab waveguide of thickness $t_{1}$, we do this for all possible directions of the TE mode in the $\mathrm{x}-\mathrm{z}$ plane. For each width, the correct value of $n_{e f f, T E}^{\text {core }}$ is 

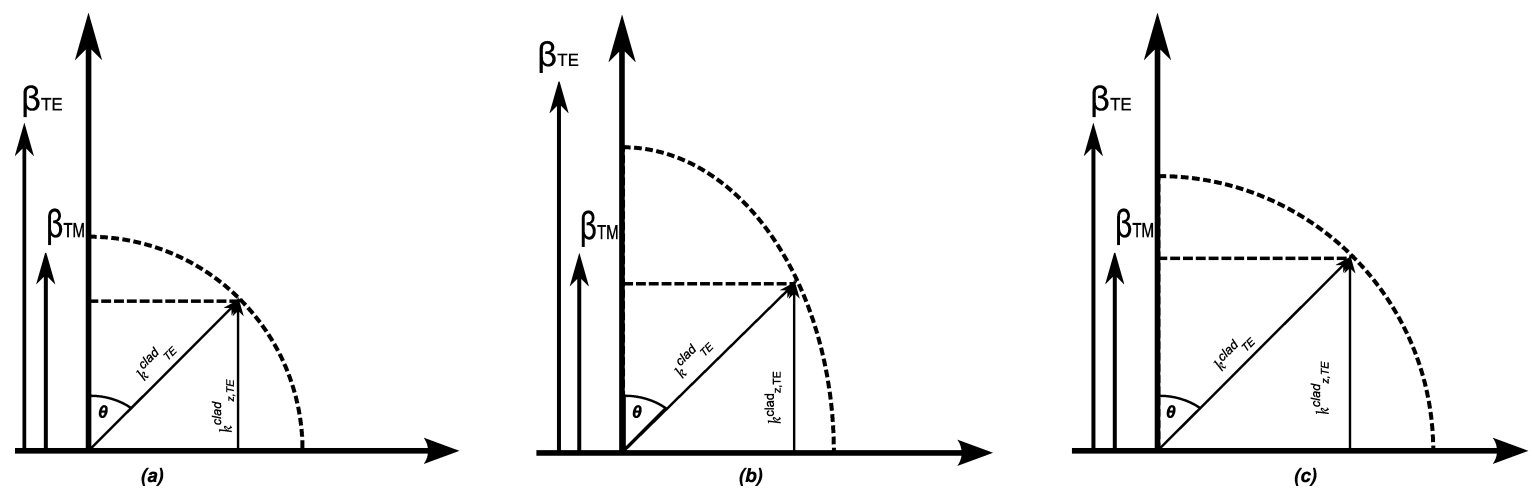

Fig. 4. Phase matching diagrams for various upper cladding configurations. (a) Air cladding. (b) LC cladding, LC oriented along x-axis; option 1. (c) LC cladding, LC oriented along the y-axis; option 2. All drawings are not to scale.

obtained by solving (4) for self consistency.

$$
\cos (\theta)=\frac{N_{e f f, T M}(W)}{n_{e f f, T E}^{\text {core }}(\theta)}
$$

For each waveguide width we now have corresponding $n_{e f f, T E}^{(c o r e)}$ and $N_{e f f, T M}$. We can make a guess of the waveguide width for which we have the first minimum in leakage loss, by plugging these values into (2) and looking for the combination of values which yield an $\mathrm{m}$ closest to 1 . We perform these calculations for each type of upper cladding. The results are tabulated below.

\begin{tabular}{cccc}
\hline$\Delta t[n m]$ & $W_{A i r}[n m]$ & $W_{L C, x}[n m]$ & $W_{L C, y}[n m]$ \\
\hline 15 & 708 & 759 & 794 \\
70 & 694 & 751 & 819 \\
\hline
\end{tabular}

Table 1. Waveguide width for which $\mathrm{m}$ in (2) is closest to unity. This gives a guess of the width at which the first minimum in leakage loss would occur. The step height for each geometry is given by, $\Delta t=t_{1}-t_{2}$.

We now turn our attention to calculating actual loss values for the geometries considered earlier. In order to do this, we employ full vectorial finite element simulations. The intent here is to verify that we indeed have tuning and ascertain the extent this tuning. We use the fully anisotropic finite element based mode-solver in COMSOL Multiphysics. We consider waveguide widths between $500 \mathrm{~nm}$ and $2500 \mathrm{~nm}$. We simulate the two cases considered earlier i.e. (i) $t_{1}=205 \mathrm{~nm}, t_{2}=190 \mathrm{~nm}$, and (ii) $t_{1}=220 \mathrm{~nm}, t_{2}=150 \mathrm{~nm}$. The refractive indices of the silicon core and silica cladding of the waveguide are $n_{\text {core }}=3.475$ and $n_{\text {clad }}=1.444$ respectively, and the operating wavelength is $1.55 \mu \mathrm{m}$.

Case one corresponds to the geometry considered in previous works [5-9] and the second case corresponds to a geometry which is in the process of being fabricated. The simulation results for case one are shown in fig. 5 below.

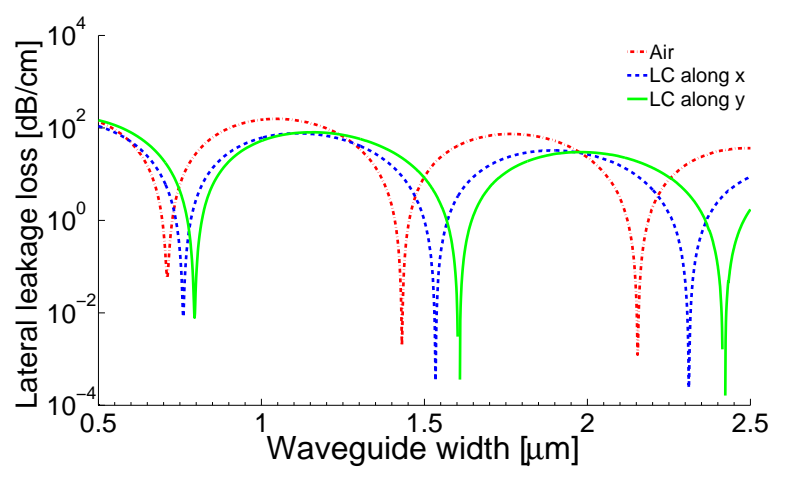

Fig. 5. Leakage loss of the fundamental TM mode for a waveguide with $t_{1}=205 \mathrm{~nm}, t_{2}=190 \mathrm{~nm}$. The loss minima occur at $711 \mathrm{~nm}, 1431 \mathrm{~nm}$ and $2154 \mathrm{~nm}$. When LC oriented along the $\mathrm{x}$-axis is used as cladding the loss minima shift to $760 \mathrm{~nm}, 1534 \mathrm{~nm}$ and $2311 \mathrm{~nm}$. After switching the LC, the loss minima shift to $795 \mathrm{~nm}, 1609 \mathrm{~nm}$ and $2423 \mathrm{~nm}$.

The simulation for an air cladding (red curve) is in excellent agreement with previous results [7]. The loss minima occur at waveguide widths of $711 \mathrm{~nm}, 1431 \mathrm{~nm}$, and $2154 \mathrm{~nm}$. When we use LC in the cladding of the waveguide, the loss minima are shifted as expected. As mentioned before, we start out with a situation in which the LC in the cladding is oriented along the $\mathrm{x}$-axis and then transition to one in which it is oriented along the y-axis. For a waveguide with LC upper cladding, the lateral leakage loss still follows a cyclic pattern. The waveguide width at which the minimum in the leakage loss occurs can be tuned by at least $35 \mathrm{~nm}$; from $760 \mathrm{~nm}$ to $795 \mathrm{~nm}$. According to our simulations, we can change the leakage loss at a width of $795 \mathrm{~nm}$, from $2 \mathrm{~dB} / \mathrm{cm}$ to about $0 \mathrm{~dB} / \mathrm{cm}$ by switching the LC from being oriented along the $\mathrm{x}$-axis to the $\mathrm{y}$-axis respectively.

Case two is for a structure which is in the process of being fabricated and for which we intend to perform loss measurements. Here the step height is more than doubled compared to the previous case. We expect a larger modulation of the leakage loss for a fixed waveg- 


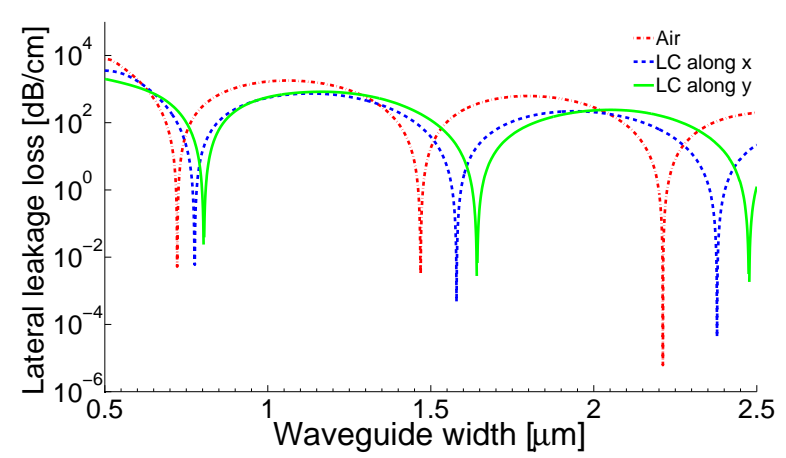

Fig. 6. Leakage loss of the fundamental TM mode for a waveguide with $t_{1}=220 \mathrm{~nm}, t_{2}=150 \mathrm{~nm}$. The loss minima occur at $722 \mathrm{~nm}, 1469 \mathrm{~nm}$ and $2212 \mathrm{~nm}$. When LC oriented along the $\mathrm{x}$-axis is used as cladding the loss minima shift to $776 \mathrm{~nm}, 1579 \mathrm{~nm}$ and $2378 \mathrm{~nm}$. After switching the LC, the loss minima shift to $804 \mathrm{~nm}, 1642 \mathrm{~nm}$ and $2476 \mathrm{~nm}$.

uide width using the LC cladding. The lateral leakage loss curves for this case are shown in fig. 6. We notice that the maximum of the leakage loss has increased by about one order of magnitude as expected. For air cladding, the loss minima occur at widths of $722 \mathrm{~nm}$, $1469 \mathrm{~nm}$ and $2212 \mathrm{~nm}$. When an LC cladding is used, we predict that it would be possible to shift the position of the leakage loss minima by $28 \mathrm{~nm}$; from a width of $776 \mathrm{~nm}$ to $804 \mathrm{~nm}$. This time for an $804 \mathrm{~nm}$ wide waveguide, the leakage loss can be changed from $19 \mathrm{~dB} / \mathrm{cm}$ to $0 \mathrm{~dB} / \mathrm{cm}$ by switching the LC from being oriented along the $\mathrm{x}$-axis to the $\mathrm{y}$-axis respectively. The change in leakage loss for case 2 is about 10 times larger than that for case 1 . This large change in leakage loss makes an experimental realization very feasible.

\begin{tabular}{cccc}
\hline$\Delta t[\mathrm{~nm}]$ & $W_{\text {Air }}[\mathrm{nm}]$ & $W_{L C, x}[\mathrm{~nm}]$ & $W_{L C, y}[\mathrm{~nm}]$ \\
\hline 15 & 711 & 760 & 795 \\
70 & 722 & 776 & 804 \\
\hline \hline 15 & $0.4 \%$ & $0.1 \%$ & $0.1 \%$ \\
70 & $4.0 \%$ & $3.3 \%$ & $1.8 \%$ \\
\hline
\end{tabular}

Table 2. Upper part, waveguide widths at which the first minimum in the loss curve occurs. Lower part, error between the predicted widths in table 1 and those in the upper part of this table. The step height for each geometry is given by, $\Delta t=t_{1}-t_{2}$.

Table 2 summarizes the values of the waveguide width at which the first minimum in the loss curves in figures 5 and 6 occur. We can now determine the accuracy of our earlier guess. For case 1, our guess is excellent; it is accurate to within $0.4 \%$ in the worst case i.e. air cladding. On the other hand, for case 2 , the guess is not that good; in the best case (LC oriented along the yaxis in the cladding), it is only accurate to within $1.8 \%$. This discrepancy is due to the difference in step-height between both cases. The accuracy of our guess deteriorates with increasing step height. We confirm this by performing simulations for waveguide geometries with step heights between $15 \mathrm{~nm}$ and $70 \mathrm{~nm}$.

\section{Wavelength dependence of the leakage loss}

From (2), it is evident that for a fixed waveguide width, the lateral leakage loss is also wavelength dependent. This has already been investigated for bent shallowetched waveguides [7]. In what follows, we look at the wavelength dependence of the lateral leakage loss when an LC cladding is used as the upper cladding of a shallow-etched waveguide.

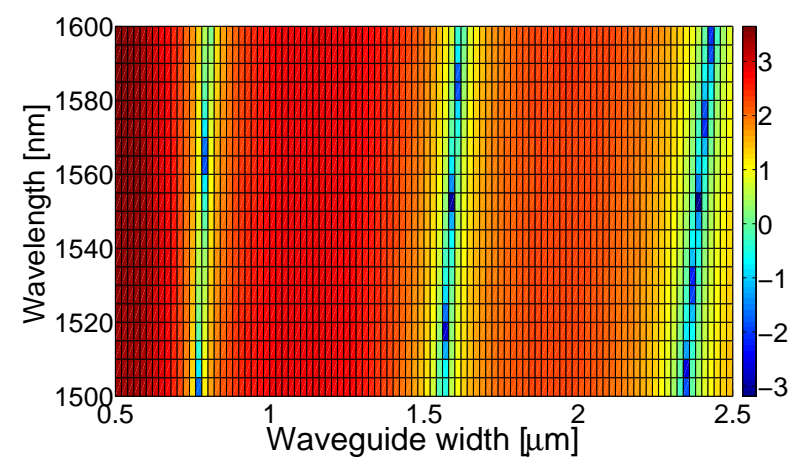

Fig. 7. Leakage loss of the fundamental TM mode for a waveguide with $t_{1}=220 \mathrm{~nm}, t_{2}=150 \mathrm{~nm}$ and LC cladding. The LC is oriented along the $\mathrm{x}$-axis. The color map is the logarithm (base 10) of the leakage loss.

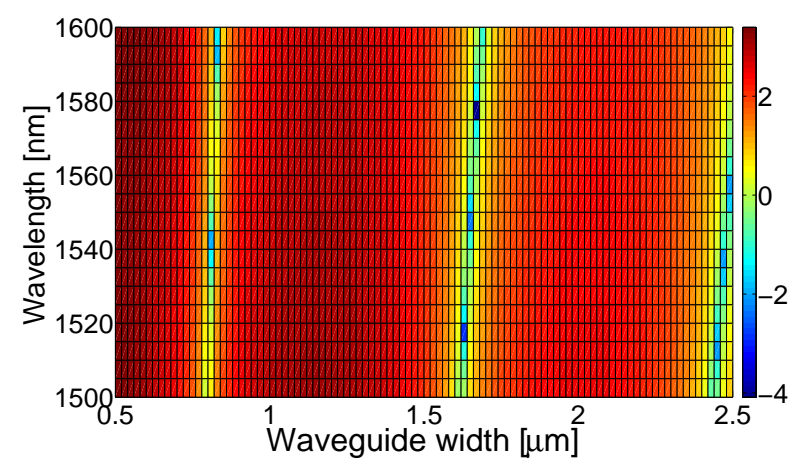

Fig. 8. Leakage loss of the fundamental TM mode for a waveguide with $t_{1}=220 \mathrm{~nm}, t_{2}=150 \mathrm{~nm}$ and LC cladding. The LC is oriented along the y-axis. The color map is the logarithm (base 10) of the leakage loss.

We perform the same simulations as in the previous section, only this time we vary the wavelength from 1.5 $\mu \mathrm{m}$ to $1.6 \mu \mathrm{m}$. For simplicity, we neglect the dispersion of the LC over this wavelength range. Figures 7 and 8 show the variation of the lateral leakage loss with both 
the waveguide width and the wavelength. The logarithm to the base 10 of the lateral leakage loss is used as the color map for these 2D plots. Overall we notice a significantly stronger dependence of the leakage loss on the waveguide width than on the wavelength. Nevertheless, for the range of waveguide widths close to the leakage loss minima, there is a strong dependence on the wavelength. In this range of widths the leakage loss varies by about $10 \mathrm{~dB} / \mathrm{cm}$ on average with the wavelength. This is an important effect which should be taken into account in practice.

\section{Discussion}

In the previous sections, we made some interesting predictions about how the leakage loss of a shallow-etched SOI waveguide with upper LC cladding would change if the $\mathrm{LC}$ is switched. We now proceed to discuss factors which could limit the predicted change in leakage loss. Accordingly, we address the effect of additional losses from the LC layer and waveguide line width variation on the change in lateral leakage loss of the waveguide.

The LC upper cladding layer can induce additional losses from the waveguide. These can either be scattering or absorption losses. In the nematic state and for the wavelength range considered, the scattering loss can be 2 orders of magnitude stronger than the absorption loss [20]. Scattering losses arise from a lack of homogeneity in the alignment of the LC molecules above the waveguide. This loss can be reduced by forcing all the molecules in the LC layer above the waveguide to be aligned in a particular direction [14]. If a voltage is then applied to the LC layer, the change in orientation would be homogeneous thus reducing scattering losses once more. Absorption losses on the other hand are intrinsic to the LC material and would vary depending on the kind of LC used. For E7, the total optical loss (i.e. scattering and absorption) is about $1 \mathrm{~cm}^{-1}$ at $1550 \mathrm{~nm}$ [20]. This corresponds to the imaginary part of the refractive index proportional to $10^{-5}$. We added this loss to the LC cladding in our simulation. The result is an increase of about $2.5 \mathrm{~dB} / \mathrm{cm}$ in lateral leakage loss compared to the case where the LC has no loss. Although the LC layer increases the total loss of the waveguides, the change in leakage loss remains the same. For case 2, and a waveguide width of $804 \mathrm{~nm}$, the leakage loss for LC oriented along the $\mathrm{x}$ and $\mathrm{y}$ axes is $21.7 \mathrm{~dB} / \mathrm{cm}$ and $2.5 \mathrm{~dB} / \mathrm{cm}$ respectively. Giving a change in loss of 19.2 $\mathrm{dB} / \mathrm{cm}$. We zoom in around waveguide widths between $750 \mathrm{~nm}$ and $820 \mathrm{~nm}$ and plot the leakage loss for $\mathrm{LC}$ with and without loss. This plot is shown in figure 9 below.

Selvaraja et al [21] report SOI waveguide line-width uniformity $<0.6 \mathrm{~nm}$ within a chip and $<2 \mathrm{~nm}$ between chips in experiments. Let us assume the worst case. If the target waveguide width is $804 \mathrm{~nm}$ but we get either $806 \mathrm{~nm}$ or $802 \mathrm{~nm}$, then the change in loss for LC reorienting from being aligned along $\mathrm{x}$ to being aligned along y would be $22 \mathrm{~dB} / \mathrm{cm}$ and $16.9 \mathrm{~dB} / \mathrm{cm}$ respectively. What's more even if the target waveguide width is $5 \mathrm{~nm}$ off i.e. $809 \mathrm{~nm}$ or $799 \mathrm{~nm}$, the change in loss would

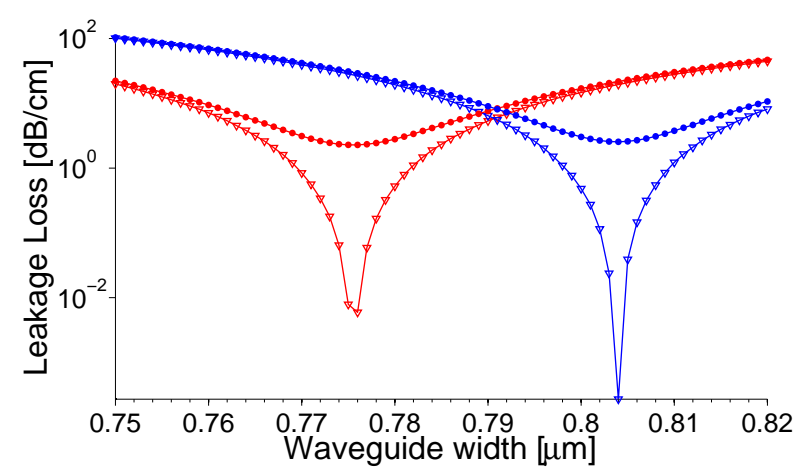

Fig. 9. Leakage loss of the fundamental TM mode for a waveguide with $t_{1}=220 \mathrm{~nm}, t_{2}=150 \mathrm{~nm}$. Curves for $\mathrm{LC}$ with loss (circles) and without loss (triangles) are shown. The red and blue curves are for LC oriented along the $\mathrm{x}$ and $\mathrm{y}$ axes respectively.

still be $25.6 \mathrm{~dB} / \mathrm{cm}$ and $12.8 \mathrm{~dB} / \mathrm{cm}$ respectively. Consequently, our prediction of a lateral leakage loss tuning range of $28 \mathrm{~nm}$ is indeed realizable.

\section{Conclusion}

We propose a method to tune the lateral leakage loss of the TM-like mode in shallow-etched SOI waveguides. We consider a shallow-etched waveguide with LC upper cladding. The effects of the LC cladding on the modes of the waveguide are explained. It is shown that the lateral leakage loss can be modulated by changing the orientation of the LC in the cladding. This change in orientation can be obtained by the application of a moderate voltage. Two different waveguide structures are considered and the lateral leakage loss is shown to increase with increasing step height. We predict that the largest modulation of the leakage loss can be observed by having a structure in which the LC is initially oriented along the $\mathrm{x}$-axis and subsequently oriented along the $\mathrm{y}$-axis. We show that our proposal is an electrically controllable switch for the leakage loss of a shallow-etched waveguide with a particular width. We also discuss the most important factors which could limit the predictions we make for change in leakage loss and come to the conclusion that the predicted change in leakage loss is experimentally achievable.

\section{Acknowledgments}

This research was supported by the Interuniversity Attraction Poles program of the Belgian Scientific Policy office under grant IAP P7-35 "photonics@be".

\section{References}

[1] G. Celler and S. Cristoloveanu, "Frontiers of silicon-oninsulator," J. Appl. Phys., vol. 93, no. 9, pp. 4955-4978, 2003.

[2] P. Dong, W. Qian, S. Liao, H. Liang, C. Kung, N. Feng, R. Shafiiha, J. Fong, D. Feng, A. krishnamoorthy, and M. Asghari, "Low loss shallow-ridge silicon waveguides," Opt. Express, vol. 18, no. 14, pp. 14474-14479, 2010. 
[3] M. Webster, R. Pafchek, G. Sukumaran, and T. Koch, "Low-loss quasi-planar ridge waveguides formed on thin silicon-on-insulator," Appl. Phys. Lett., vol. 87, no. 23, 2005.

[4] R. Pafchek, R. Tummidi, J. Li, M. Webster, E. Chen, and T. Koch, "Low-loss silicon-on-insulator shallowridge te and tm waveguides formed using thermal oxidation," Appl. Optics, vol. 48, no. 5, pp. 958-963, 2009.

[5] M. Webster, R. Pafchek, A. Mitchell, and T. Koch, "Width dependence of inherent tm-mode lateral leakage loss in silicon-on-insulator ridge waveguides," IEEE Phot. Tech. Lett., vol. 19, no. 6, pp. 429-431, 2007.

[6] T. Nguyen, R. Tummidi, T. Koch, and A. Mitchell, "Lateral leakage of tm-like mode in thin-ridge siliconon-insulator bent waveguides and ring resonators," Opt. Express, vol. 18, no. 7, 2010.

[7] M. Koshiba, K. Kakihara, and K. Saitoh, "Reduced lateral leakage losses in tm-like modes in silicon-oninsulator ridge waveguides," Opt. Lett., vol. 33, no. 17, pp. 2008-2010, 2008.

[8] K. Kakihara, K. Saitoh, and M. Koshiba, "Generalized simple theory for estimating lateral leakage loss behavior in silicon-on-insulator ridge waveguides," J. Lightwave Tech., vol. 27, no. 23, pp. 5492-5499, 2009.

[9] N. Dalvand, T. Nguyen, R. Tummidi, T. Koch, and A. Mitchell, "Thin-ridge silicon-on-insulator waveguides with directional control of lateral leakage radiation," Opt. Express, vol. 19, no. 6, pp. 5635-5643, 2011.

[10] M. Pruessner, T. Stievater, M. Ferraro, and W. Rabinovich, "Thermo-optic tuning and switching in siliconon-insulator waveguide fabry-perot microcavities," Opt. Express, vol. 15, no. 12, pp. 7557-7563, 2007.

[11] P. Dong, W. Qiang, H. Liang, R. Shafiiha, D. Feng, G. Li, J. Cunningham, A. Krishnamoorthy, and M. Asghari, "Thermally tunable silicon racetrack resonators with ultralow tuning power," Opt. Express, vol. 18, no. 19, pp. 20298-20304, 2010.

[12] J. Beeckman, K. Neyts, and P. Vanbrabant, "Liquidcrystal photonic applications," Opt. Engineering, vol. 50, no. 17, 2011.
[13] T. Wang, S. Yang, T. Chen, and B. Chen, "Wide tuning of sin microring resonators by auto-realigning nematic liquid crystal," Opt. Express, vol. 20, no. 14, pp. 1585315858, 2012.

[14] W. Cort, J. Beeckman, T. Claes, K. Neyts, and R. Baets, "Wide tuning of silicon-on-insulator ring resonators with a liquid crystal cladding," Opt. Lett., vol. 36, no. 19, pp. 3876-3878, 2011.

[15] J. Beeckman, R. James, F.A., Fernandes, W. Cort, and K. Neyts, "Calculation of fully anisotropic liquid crystal waveguide modes," J. Lightwave Tech., vol. 27, no. 21, pp. 3812-3819, 2009.

[16] S. Ertman, T. Wolinski, D. Pysz, R. Buczynski, E. Nowinski-Kruszelnicki, and R. Dabrowski, "Low-loss propagation and continuosly tunable birefringence in high-index photonic crystal fibers filled with nematic liquid crystals," Opt. Express, vol. 17, no. 21, pp. 1929819310, 2009.

[17] A. d'Alessandro, B. Bellini, D. Donisi, R. Beccherelli, and R. Asquini, "Nematic liquid crystal optical channel waveguides on silicon," IEEE J. Quantum Electron., vol. 42, pp. 1084-1090, sep 2006.

[18] A. Fratalocchi, R. Asquini, and G. Assanto, "Integrated electro-optic switch in liquid crystals," Opt. Express, vol. 13 , pp. 32-36, sep 2005.

[19] A. Oliner, S. Peng, T. Hsu, and A. Sanchez, "Guidance and leakage properties of a class of open dielectric waveguides: Part ii-new physical effects," IEEE Trans.Microw. Theory and Tech., vol. 29, pp. 855-869, sep 1981.

[20] S.T. Wu, and K.C. Lim, "Absorption and scattering measurements of nematic liquid crystals," Appl. Opt., 26:1722-1727, 1987.

[21] S. Selvaraja, W. Bogaerts, P. Dumon, D. Van Thourhout, and R. Baets, "Sub-nanometer linewidth uniformity in silicon nano-photonic waveguide devices using cmos fabrication technology," J. of selected topics in Quantum Electronics, 16(1):316-324, 2010. 